\title{
Spinecor vs exercises for adolescent idiopathic scoliosis: short term results
}

\author{
Fabio Zaina*, Claudia Fusco, Michele Romano, Alessandra Negrini, Stefano Negrini \\ From 7th International Conference on Conservative Management of Spinal Deformities \\ Montreal, Canada. 20-22 May 2010
}

\section{Introduction}

SpineCor and exercises have both results testifying their effectiveness in Adolescent Idiopathic Scoliosis (AIS) treatment. Since some years we introduced SpineCor as a treatment for patients at highest risk of bracing that we previously treated with exercises. The objective of this study is to compare the short term results of the Spinecor vs SEAS exercises for AIS.

\section{Materials and methods}

Study design: retrospective controlled study

\section{Population}

56 consecutive AIS patients from our prospective database (39 female; age $13 \pm 1$, Cobb $21 \pm 4^{\circ}$; ATR $11 \pm 4^{\circ}$ ): 28 patients ( 19 females; age $13 \pm 1$; TRACE score 6 ; Cobb angle $22 \pm 4^{\circ}$; ATR $12 \pm 4^{\circ}$, Risser 0 -3) treated by Spinecor 20/24 hours; 28 patients (20 females; age $13 \pm 1$; TRACE score 5; Cobb angle $20 \pm 4^{\circ}$; ATR $9 \pm 3^{\circ}$, Risser 0 -3) treated by SEAS exercises. The short term results of treatment were evaluated at the moment of the first Xray performed without the Spinecor $17 \pm 4$ months later. For the SEAS group the X-ray were performed after 14 \pm 4 . Patients were evaluated both clinically and radiographically before and after the treatment. Main outcome measures: $\mathrm{N}^{\circ}$ of patients who had to wear a rigid brace, TRACE (Trunk Aesthetic Clinical Evaluation), Cobb angle (changes $> \pm 4$ ), ATR (changes $> \pm 2$ ).

\section{Result}

There were no statistically significant differences between the groups at baseline even if Cobb angles, ATR and TRACE were slightly worst in SpineCor group. The $\mathrm{N}$ of patients prescribed with a rigid brace was $4(14 \%)$ in the SEAS Group vs $3(10 \%)$ in the
Spinecor, not significant (NS). The Median value of TRACE didn't change for SEAS while decreased for Spinecor ( 5 vs $4, \mathrm{p}<0.05$ ). Considering the number of patients changed $> \pm 5^{\circ} \mathrm{Cobb}$ in the SEAS Group there was $39.3 \%$ improved and $14.3 \%$ worsened vs $25 \%$ and $21.4 \%$ in SpineCor (NS). For ATR there were 50\% Improved and $50 \%$ Stable for SEAS group vs $57 \%$ and $43 \%$ for Spinecor (NS).

\section{Discussion}

There were no differences in the number of rigid braces prescribed. The Spinecor showed to be able to improved the Aesthetics (TRACE) in AIS patients while exercise maintained a stability. The number of patients clinically changed showed a similar efficacy of both treatments. The main limit of the study are the retrospective design, the small population and the short term evaluation. We can argue that with a longer follow up the Spinecor can achieve better results in terms of avoiding rigid brace prescription and curve progression.

\section{Conclusion}

Short term results showed a better efficacy of the Spinecor for Aesthetics in AIS while the efficacy on avoiding curve progression was similar to the SEAS exercises.

Published: 10 September 2010

doi:10.1186/1748-7161-5-S1-053

Cite this article as: Zaina et al: Spinecor vs exercises for adolescent idiopathic scoliosis: short term results. Scoliosis 2010 5(Suppl 1):O53. 